\title{
A Levelled Architecture for Designing Smart Cities
}

\author{
Poonam Sharma \\ Assistant Professor, Department of computer science \\ Amity University, Haryana. 122051
}

\begin{abstract}
Smart offerings are crucial element of the smart towns and the Internet of Things (IoT) ecosystems wherein the intelligence in the back of the services is obtained and stepped forward through the sensory statistics. Providing a massive amount of data facts is not usually viable; therefore, we need to consider alternative methods that include unlabeled statistics as well. In current years, deep learning has gained first-rate achievement in several application domains. It is an applicable method for IoT and smart city scenarios where automobile-generated statistics may be in part categorised via users' comments for training functions. In this paper, we suggest a deep learning mode that fits to IoT and smart city development because it consumes both classified and unlabeled facts to enhance the overall performance and accuracy of the learning agent.
\end{abstract}

Keywords: smart cities, big data, analytics, IoT, machine learning.

\section{INTRODUCTION}

The need to cope with urban improvement isn't always new. Many of the things we take with no consideration nowadays had been implemented to clear up specific troubles dealing with our towns. Examples include: skyscrapers, mass transit, waste water treatment, visitors lighting, and so on.

We now face new demanding situations as the both the wide variety and size of towns increase. These challenges are also possibilities. Beyond the blessings that cities provide to their inhabitants within the way of access to services, towns also provide a manner to fulfil the population's needs extra efficaciously and in a way that is less detrimental to the surroundings than a dispersed rural population. The main features required for designing a smart city are depicted in fig 1.

Rapid development of Internet of Things (IoT) technologies and deep learning motivated researchers and developers to suppose about new forms of smart services that extract expertise from IoT generated statistics. The scarcity of classified data is a main issue for growing such solutions mainly for IoT programs [1].

In this paper we discuss about a multi-level architecture for designing the smart cities and also some real time applications of the same.

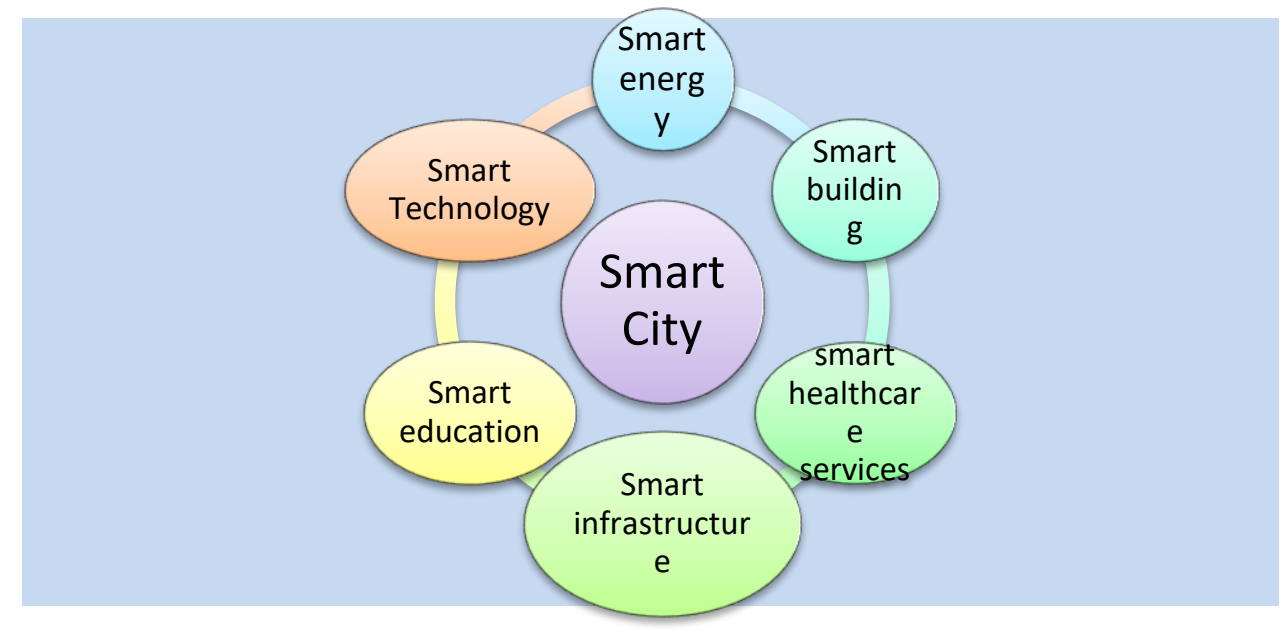

Fig 1. Smart city concepts

\section{BACKGROUND}

IoT basically stands for interconnecting the various devices with each other. Fig 2 depicts the kind of interconnections and actual applications of IoT. The data is generated from various heterogeneous sources which are nothing but sensors. The major challenge is to design a fusion system for integrating the data and use them for important purposes. 


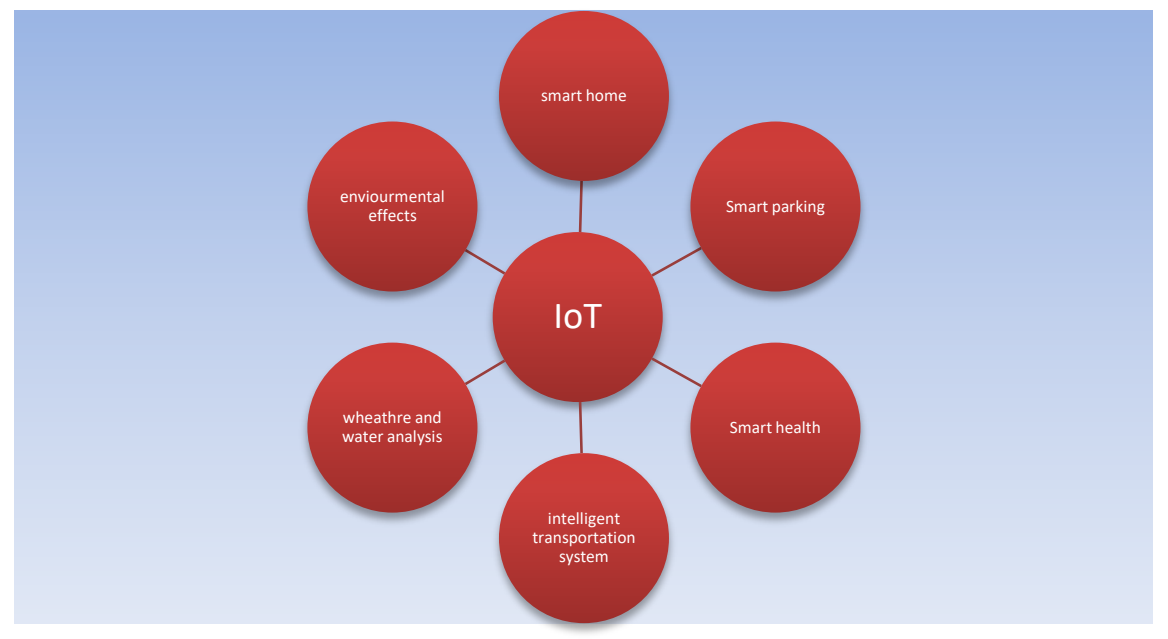

Fig 2. Application areas of IoT

There are some existing smart city projects which defined the model for designing a smart city. Like SCRIBE designed and proposed by IBM. The SCRIBE ontology is described using open requirements along with Common Alerting Protocol and the National Information Exchange Model (NIEM) and addresses the heterogeneous information issue in different Smart City domain names. Similarly, the Smart Santander project8 ambitions to evaluate the important thing building blocks of the IoT, which might be mainly the interplay and control protocol mechanisms. In the Smart Santander mission, a massive quantity of sensors may be deployed in specific cities and exploited for distinct packages. The developed check bed will assist in exploiting diverse Smart City domains such as environment tracking, traffic intensity sample monitoring and steering for drivers on available parking spaces. The City Sense project9 aimed to improve current human infrastructure and accordingly assist in presenting better services to residents with the aid of exploiting to be had resources (together with energy, water, and site visitors) in a more green manner. However, those Smart City tasks do not offer distinctive information approximately their implementation. In addition, their semantic models do not specify how they will incorporate uncertainty factors.

These aspects can be resolved by using a multilevel architecture discussed in next section.

\section{LEVELLED ARCHITECTURE FOR SMART-CITY}

As IoT is a broadband network using standard protocols and data from heterogeneous sources technology plays great role in designing such networks. The major technological components of IoT applications are.

A. Radio frequency Identification (RFID)

These systems include tags which are key position within the context of the IoT. By making use of these technologies to any involved object, it's miles viable to perform their automated identity and assign a completely unique digital identification to each object, as a way to be included in the network and related to the virtual records and carrier [2].

$B$. Wireless sensor networks

WSNs can provide unique appropriate statistics and additionally may be used in many cases inclusive of healthcare, authorities and environmental services and seismic sensing. Furthermore, WSNs may be integrated with RFID structures to gain some goals like obtaining statistics regarding the location, movement, temperature, and so on [3-5].

C. Addressing and Middleware

Because the Internet can allow a superb interconnection of humans, the present fashion in the IoT can in addition provide an interconnection of gadgets and things, in order to establish clever environments. To this quit, the capability of uniquely figuring out items is vital for favourable outcomes of the IoT. The key objective of the middleware is, indeed, to concisely combine the functionalities and verbal exchange capabilities of all concerned devices [6-7].

In the levelled architecture whole of the process of designing smart network is divided into four major categories between sensors and provided service as shown in fig 3 . 


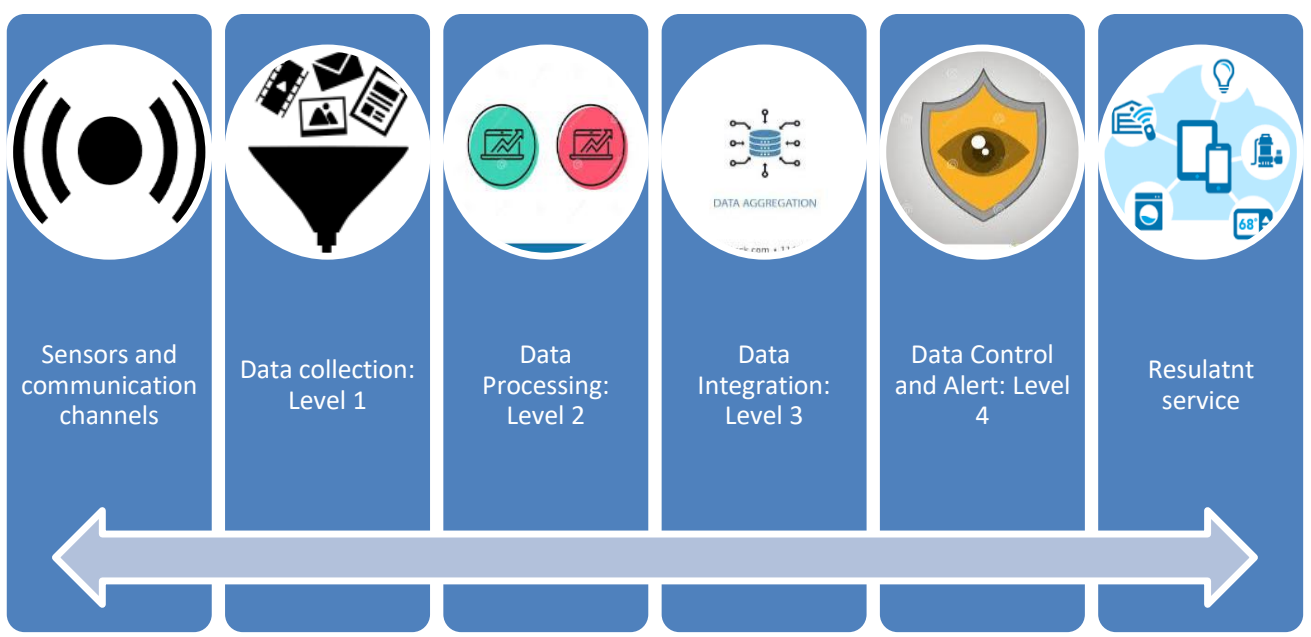

Fig 3. Levelled architecture of IoT

\section{A. Level 1: Data Collection}

At this level raw data is collected from various heterogeneous resources. This data needs to be converted into a form which can be web consumable. Some of the formats in which heterogeneous information are accumulated are csv, tweets, database schemas and text messages. The accrued formats are then processed the usage of semantic internet technology with a view to convert them right into a common format.

B. Level 2: Data Pre-processing

The major goal of this stage is to convert the accumulated heterogeneous data right into a commonplace format, e.g. Resource Description Framework (RDF). This is the maximum common way to trade statistics over the web and it facilitates heterogeneous facts sharing and integration for exceptional Smart City domain names [8]. RDF additionally allows in defining metadata approximately the assets at the web. Different software program applications can then make use of RDF statistics for intelligent reasoning operations.

\section{Level 3: Data Integration}

Semantic net technologies allow exploitation of area specific information based totally at the standards and relationships among the ones principles. Web ontology language (OWL) is used for publishing the ontologies. OWL is an RDF graph this is constructed the usage of the RDF and ontologies. It lets in the type of the individual/concepts primarily based at the training. It additionally affords exceptional varieties of residences, which can be used to form relationships between exceptional classes, specifically the Data property and Object assets.

Once information class is carried out, expertise may be further used for analytics purpose. SPARQL is an RDF question language which is used to question, retrieve and manage records/statistics stored in the RDF layout. Once the whole database is expressed within the shape of RDF triples, SPARQL enables the question and retrieval of facts in the identical layout. Therefore, this level motivates closer to low-degree statistics fusion.

D. Level 4: Data control and alert

Data acquired from previous level can be utilized by special internet packages for shrewd running conditions. The inferred records can be utilized in lots of methods such as enter/output, messaging, signals and warnings.

\section{CONCLUSION}

Implementation of the IoT infrastructures should enable a number of possibilities,first of all the very best studies motivations are defined after which some beneficial applications outlined. It is defined how each day activities can be advanced and more suitable by using using them. Also, the challenges which get up when enforcing the IoT device had been very well explained. For overcome these challenges a levelled architecture is discussed which can lower down the dependencies among different tasks and hence can increate the connectivity and accuracy of the network. Future work is deliberate to carry out experiments on the thoughts mentioned, which incorporates discovering real-time heterogeneous data, presenting a common semantic expertise model, using Dempster-Shafer methods for combining sensor records and for reasoning, and defining information interoperability and scalability factors in the discussed architecture.

\section{REFERENCES}

[1] Poonam Sharma, Akansha Singh, " Era of deep neural networks: A review" ICCCNT 2017, pp. 1-5, 2017.

[2] A.K. Evangelos, D.T. Nikolaos, and C.B. Anthony, "Integrating RFIDs and smart objects into a Unified Internet of Things architecture," Advances in Internet of Things, vol. 1, pp. 5-12, 2011.

[3] "Six Technologies with Potential Impacts on US Interests Out to 2025," Disruptive civil technologies, 2008. 
[4] A. Alamri, W.S. Ansari, M.M. Hassan, M.S. Hossain, A. Alelaiwi, and M.A. Hossain, "A survey on sensor-Cloud: architecture, applications, and approaches," Int. J. Distrib. Sens. Netw., vol. 2013, pp. 1-18, 2013.

[5] M. Shafie-khah, M. Kheradmand, S. Javadi, M. Azenha, J.L.B. de Aguiar, J. Castro-Gomes, P. Siano, and J.P.S. Catalão, "Optimal behavior of responsive residential demand considering hybrid phase change materials," Appl. Energy, vol. 163, pp. 81-92, 2016.

[6] J. Gubbi, R. Buyya, S. Marusic, and M. Palaniswami, "Internet of Things (IoT): A vision, architectural elements, and future directions," Future Gener. Comput. Syst., vol. 29, pp. 1645-1660, 2013.

[7] L. Atzori, A. Iera, and G. Morabito, "The internet of things: a survey," Comput. Netw, vol. 54, pp. 2787-2805, 2010.

[8] Resource Description Framework (RDF) Model and Syntax Specification. Available at: http://www.w3.org/TR/PRrdf-syntax/. 